\title{
The Role of Sirtuin 1 in Palmitic Acid-Induced Endoplasmic Reticulum Stress in Cardiac Myoblasts
}

\author{
Hsiang-Yu Yang ${ }^{1,2,3}{ }^{-}$, Jhao-Ying Chen ${ }^{3}{ }^{1}$, , Yen-Nien Huo ${ }^{4}$, Pei-Ling $\mathrm{Yu}^{3}$, Pei-Zhen Lin ${ }^{3}$, Shih-Che Hsu ${ }^{5}{ }^{(0)}$, \\ Shih-Ming Huang ${ }^{3} \mathbb{D}$, Chien-Sung Tsai ${ }^{1,6, *}$ and Chih-Yuan Lin ${ }^{1,3, *}$
}

check for

updates

Citation: Yang, H.-Y.; Chen, J.-Y.;

Huo, Y.-N.; Yu, P.-L.; Lin, P.-Z.; Hsu,

S.-C.; Huang, S.-M.; Tsai, C.-S.; Lin,

C.-Y. The Role of Sirtuin 1 in Palmitic

Acid-Induced Endoplasmic

Reticulum Stress in Cardiac

Myoblasts. Life 2022, 12, 182.

https://doi.org/10.3390/

life12020182

Academic Editor: Yongseek Park

Received: 30 December 2021

Accepted: 24 January 2022

Published: 26 January 2022

Publisher's Note: MDPI stays neutral with regard to jurisdictional claims in published maps and institutional affiliations.

Copyright: (C) 2022 by the authors. Licensee MDPI, Basel, Switzerland. This article is an open access article distributed under the terms and conditions of the Creative Commons Attribution (CC BY) license (https:// creativecommons.org/licenses/by/ $4.0 /)$.
1 Division of Cardiovascular Surgery, Department of Surgery, Tri-Service General Hospital, National Defense Medical Center, Taipei 114, Taiwan; alfie0314@mail.ndmctsgh.edu.tw

2 Graduate Institute of Life Sciences, National Defense Medical Center, Taipei 114, Taiwan

3 Department of Biochemistry, National Defense Medical Center, Taipei 114, Taiwan; illy49947125@gmail.com (J.-Y.C.); linda81510@gmail.com (P.-L.Y.); xpeachcj8a8@gmail.com (P.-Z.L.); shihming7102@gmail.com (S.-M.H.)

4 Graduate Institute of Medical Sciences, College of Medicine, Taipei Medical University, Taipei 114, Taiwan; poooooh_1988@hotmail.com

5 CVie Therapeutics Ltd., Taipei 114, Taiwan; evanhsu6938@gmail.com

6 Department and Graduate Institute of Pharmacology, National Defense Medical Center, Taipei 114, Taiwan

* Correspondence: sung1500@mail.ndmctsgh.edu.tw (C.-S.T.); linrock@ms26.hinet.net (C.-Y.L.); Tel.: +886-2-8792-7212 (C.-Y.L.); Fax: +886-2-8792-7376 (C.-Y.L.)

\begin{abstract}
Background: Lipotoxicity causes endoplasmic reticulum (ER) stress, leading to cell apoptosis. Sirtuin 1 (Sirt1) regulates gene transcription and cellular metabolism. In this study, we investigated the role of Sirt1 in palmitate-induced ER stress. Methods: Both H9c2 myoblasts and heart-specific Sirt1 knockout mice fed a palmitate-enriched high-fat diet were used. Results: The high-fat diet induced C/EBP homologous protein (CHOP) and activating transcription factor 4 (ATF4) expression in both Sirt1 knockout mice and controls. The Sirt1 knockout mice showed higher CHOP and ATF4 expression compared to those in the control. Palmitic acid (PA) induced ATF4 and $\mathrm{CHOP}$ expression in $\mathrm{H} 9 \mathrm{c} 2$ cells. PA-treated H9c2 cells showed decreased cytosolic $\mathrm{NAD}^{+} / \mathrm{NADH}$ alongside reduced Sirt1's activity. The H9c2 cells showed increased ATF4 and CHOP expression when transfected with plasmid encoding dominant negative mutant Sirt1. Sirt1 activator SRT1720 did not affect CHOP and ATF4 expression. Although SRT1720 enhanced the nuclear translocation of ATF4, the extent of the binding of ATF4 to the CHOP promoter did not increase in PA treatedH9c2 cells. Conclusion: PA-induced ER stress is mediated through the upregulation of ATF4 and CHOP. Cytosolic $\mathrm{NAD}^{+}$concentration is diminished by PA-induced ER stress, leading to decreased Sirt1 activity. The Sirt1 activator SRT1720 promotes the nuclear translocation of ATF4 in PA-treated $\mathrm{H} 9 \mathrm{c} 2$ cells.
\end{abstract}

Keywords: palmitate; endoplasmic reticulum stress; sirtuin 1

\section{Introduction}

Cardiac lipotoxicity, featuring toxic lipid accumulation in the heart, plays a pathological role in the development of obesity induced cardiovascular diseases [1]. The effect of obesity epidemic and dietary fat intake on the development of cardiovascular disease and progression of heart failure has been receiving greater attention in recent years [2,3]. Saturated fatty acids, especially $16-\mathrm{C}$ palmitate, are reported to be more lipotoxic than unsaturated fatty acids in cardiomyocytes [4]. A major mechanism underlying the palmitateinduced cardiomyocyte dysfunction is endoplasmic reticulum (ER) stress, which may promote cell death [5].

ER stress results from an excessive accumulation of misfolded proteins within the ER and triggers a compensatory mechanism, the unfolded protein response (UPR), intended to 
modulate ER stress and restore ER homeostasis [6]. ER transmembrane proteins, including PKR-like ER kinase (PERK), activating transcription factor 6 (ATF6), and inositol-requiring enzyme 1 (IRE1), are responsible for UPR initiation. The UPR may switch to a proapoptotic signaling pathway to terminate the cell dysfunction. In the apoptosis pathways, activating transcription factor 4 (ATF4) plays a crucial role because it drives the transcription of several apoptosis genes, including a proapoptotic one, $\mathrm{C} / \mathrm{EBP}$ homologous protein $(\mathrm{CHOP})$, also known as DNA damage-inducible transcript 3 protein.

Sirtuin 1 (Sirt1) is a member of the sirtuin family and is an $\mathrm{NAD}^{+}$-dependent enzyme performing the deacetylation of target substrates by hydrolyzing $\mathrm{NAD}^{+}$to produce a deacetylated substrate, acetyl-ADP-ribose, and nicotinamide. Furthermore, Sirt1 is capable of deacetylating acetyl-lysine in histones, such as $\mathrm{H3K} 9 \mathrm{Ac}$, for gene transcription modulation. As is known, Sirt1 plays an important part in the regulation of the cellular metabolism, inflammation, the cell cycle, and DNA repair [7]. An impairment of cardiac Sirt1 signaling is reported to contribute to the pathogenesis of cardiovascular diseases [8-10]. Additionally, Sirt1 has been shown to provide cardio protection against ER stress-induced cell death through eIF2 $\alpha$ deacetylation in a Sirt1 knockout mouse model [11]. However, the role of Sirt1 in saturated fatty acid, such as palmitate, induced ER stress is still not fully understood [12].

The aim of the present study was to investigate the mechanism of palmitate-induced ER stress in cardiomyocytes and to determine the role of Sirt1. We hypothesized that Sirt1 provides protection against palmitate and a high fat diet induces ER stress in cardiac myocytes. Here, we used H9c2 myoblasts and mice with a heart-specific Sirt1 exon-4 knockout fed a palmitate-enriched high-fat diet (HFD) to identify the signaling pathway involved in palmitic acid (PA)-induced ER stress. We tested whether Sirt1 expression is affected by PA-induced ER stress and explored the possible protective effects of Sirt1. We hope that our current findings provide a novel insight into PA-induced ER stress and into the function of Sirt1 in cardiomyocytes.

\section{Methods}

\subsection{Genetically Modified and HFD Mouse Models}

Animal experiments were all conducted with the approval of the Institutional Animal Care and Use Committee (IACUC, permit No. 19-364) of the National Defense Medical Center (Taipei, Taiwan) and in accordance with the National Institutes of Health guidelines, "Guide for the Care and Use of Laboratory Animals", on manipulations with experimental animals. The study was carried out in compliance with the ARRIVE guidelines.

The mice with the heart-specific Sirt1 exon- 4 knockout (Sirt1 ${ }^{-/}$) were created by crossing Sirt $1^{\text {flox/flox }}$ mice (controls purchased from Jackson Laboratory) with mice carrying $\alpha$-MHC (myosin heavy chain) promoter-driven Cre in a C57BL/6J background ( $\alpha$-MHCCre mice, courtesy of Prof. M. Schneider, Imperial College London) and are currently in use in the laboratory [13]. Six-week-old mice were separately fed either a standard diet (SD) (10\% kcal fat, D17071303i, Research Diets, New Brunswick, NJ, USA) or a palmitateenriched HFD (60\% kcal fat, D16042106i, Research Diets, New Brunswick, NJ, USA) for 8 weeks and then were euthanized to collect the hearts for subsequent experiments. The animals were kept at a temperature of $21 \pm 1{ }^{\circ} \mathrm{C}$ on a controlled 12:12 h light-dark cycle with ad libitum access to deionized drinking water before the experiments.

\subsection{Cardiomyocyte Isolation}

Ventricular myocytes were enzymatically dissociated as previously described [14], with modifications. Briefly, mice were euthanized using a mixture of Zoletil 50 and xylazine, and the hearts were excised and cannulated via the aorta to a Langendorff perfusion system at $37{ }^{\circ} \mathrm{C}$. Each heart was first perfused with normal Tyrode's solution $(137 \mathrm{mM} \mathrm{NaCl}$, $1.8 \mathrm{mM} \mathrm{CaCl}_{2}, 0.5 \mathrm{mM} \mathrm{MgCl} 2,5.4 \mathrm{mM} \mathrm{KCl}, 10 \mathrm{mM}$ glucose, and $10 \mathrm{mM} \mathrm{HEPES}[\mathrm{pH}$ adjusted to 7.4 with $\mathrm{NaOH}])$ for $10 \mathrm{~min}$ and digested with a $\mathrm{Ca}^{2+}$-free solution $(120 \mathrm{mM}$ $\mathrm{NaCl}, 5.4 \mathrm{mM} \mathrm{KCl}, 1.2 \mathrm{mM} \mathrm{MgSO}_{4}, 1.2 \mathrm{mM} \mathrm{KH}_{2} \mathrm{PO}_{4}, 6 \mathrm{mM}$ HEPES, $10 \mathrm{mM}$ glucose, and 
$10 \mathrm{mM}$ taurine [pH adjusted to 7.4 using $\mathrm{NaOH}]$ ) containing $1 \mathrm{mg} / \mathrm{mL}$ collagenase (Type I, Sigma-Aldrich, St. Louis, MO, USA) and $0.06 \mathrm{mg} / \mathrm{mL}$ proteinase (type XIV, Sigma-Aldrich, St. Louis, MO, USA). After the perfusion, the heart was disconnected from the cannula, cut into small pieces, and gently triturated with a plastic transfer pipette, and the homogenate was filtered through a nylon mesh. The dissociated cells were stored in normal Tyrode's solution at $20-22{ }^{\circ} \mathrm{C}$. Rod-shaped cells with clear striations and no granulation were used within $6-8 \mathrm{~h}$ for all the experiments.

\subsection{H9c2 Culture and Plasmid Transfection}

The H9c2 rat myoblast cell line (BCRC60096) was purchased from the Bioresource Collection and Research Center of the Food Industry Research and Development Institute (Taipei, Taiwan). The cells were cultured in Dulbecco's modified Eagle's medium (DMEM) supplemented with $10 \%$ of fetal bovine serum, $150 \mathrm{U} / \mathrm{mL}$ penicillin, and $150 \mathrm{mg} / \mathrm{mL}$ streptomycin. The cells were incubated at $37^{\circ} \mathrm{C}$ in $5 \% \mathrm{CO}_{2} / 95 \%$ air. Confluent cells were detached with a $0.05 \%$ trypsin $/ 0.02 \%$ EDTA solution and subcultured in 6-well culture plates to obtain the second passage as previously described [15]. To check the protein levels and mRNA expression of CHOP and ATF4 induced by PA, the H9c2 cells were treated with PA with concentrations ranging from 0 to $200 \mu \mathrm{M}$ for $24 \mathrm{~h}$. cDNA sequences of full-length mutant Sirt1 (H363Y) were obtained from Addgene (Cambridge, MA, USA) [16,17]. cDNAs of mutant Sirt1 (H363Y) were cloned into the pSG5.HA vector [18]. On the following day, the cells were plasmid-transfected by means of the Lipofectamine 3000 Reagent (Thermo Fisher Scientific Co., Carlsbad, CA, USA) according to the manufacturer's instructions. After $24 \mathrm{~h}$ transfection, the H9c2 cells were treated with $150 \mu \mathrm{M}$ PA for $12 \mathrm{~h}$.

\subsection{Immunoblotting Analysis}

Ventricular tissues were homogenized in FastPrep-24 5G (MP Biomedicals, Irvine, CA, USA) with RIPA buffer (100 mmol/L Tris- $\mathrm{HCl} \mathrm{pH} \mathrm{8.0,} 0.1 \%$ of sodium dodecyl sulfate, $1 \%$ of Triton X-100, and $150 \mathrm{mmol} / \mathrm{L} \mathrm{NaCl}$ ) containing a protease inhibitor cocktail (Roche, Basel, Switzerland), followed by centrifugation at $15,000 \mathrm{rpm}$ for $15 \mathrm{~min}$ at $4{ }^{\circ} \mathrm{C}$. The protein content was determined in the supernatants, according to the DC Protein Assay instruction manual (Bio-Rad, Hercules, CA, USA). The H9c2 cells were lysed in RIPA buffer (100 mM Tris- $\mathrm{HCl} \mathrm{pH} 8.0,150 \mathrm{mM} \mathrm{NaCl}, 0.1 \%$ of SDS, and $1 \%$ of Triton X-100) and then centrifuged for $15 \mathrm{~min}$ at $15,000 \mathrm{rpm}$ at $4{ }^{\circ} \mathrm{C}$. The protein extracts from heart tissue and from cultured $\mathrm{H} 9 \mathrm{c} 2$ cells were separated by SDS polyacrylamide gel electrophoresis and then transferred to polyvinylidene difluoride membranes (Merck Millipore, Burlington, MA, USA), which were incubated with the following antibodies: anti- $\alpha$-Tubulin $(1: 10,000$, Proteintech, Manchester, UK), anti-GAPDH (1:10,000, Proteintech, UK), anti-CHOP (1:800, Cell Signaling Technology, Danvers, MA, USA), anti-ATF4 (1:800, Santa Cruz Biotechnology, Dallas, TX, USA), anti-Sirt1 (1:800, Merck Millipore, Burlington, MA, USA), and anti-acH3K9 (1:1000, Merck, Kenilworth, NJ, USA). Subsequently, the membranes were incubated with anti-mouse (Santa Cruz Biotechnology, Dallas, TX, USA, sc-2056) or anti-rabbit (Santa Cruz Biotechnology, Dallas, TX, USA, sc-2004) secondary IgG antibodies at a dilution of 1:10,000. Immunoreactive proteins were detected via enhanced chemiluminescence (GE Healthcare, Chicago, IL, USA) and were then quantified in the ImageJ software 1.5 (NIH, Bethesda, MD, USA).

\subsection{RT-PCR Analysis}

Total RNA was extracted from heart tissue and cultured H9c2 cells using the Total RNA reagent (Bioman, New Taipei City, Taiwan). Next, $1 \mu \mathrm{g}$ of total RNA was reverse-transcribed using the Moloney murine leukemia virus (MMLV) reverse transcriptase (Epicentre Biotechnologies, Madison, WI, USA) as per the manufacturer's instructions. The resultant cDNA was quantified by quantitative RT-PCR in an Illumina ECO ${ }^{\mathrm{TM}}$ Real-Time PCR system. Cycle threshold $\left(C_{t}\right)$ values for target mRNAs were normalized to the housekeeping gene GAPDH, and the relative gene expression was calculated by the $2^{-\Delta \Delta \mathrm{Ct}}$ method. 
Primer sequences were as follows: GAPDH forward 5'-GGATACTGAGAGCAAGAG AGAGG-3' and reverse 5'-TCCTGTTGTTATGGGGTCTGG-3', CHOP forward $5^{\prime}$-CCAGCA GAGGTCACAAGCAC-3' ${ }^{\prime}$ and reverse $5^{\prime}$-CGCACTGACCACTCTGTTTC-3' ${ }^{\prime}$, and ATF4 forward 5'-CCTGACTCTGCTGCTTATATTACTCTAAC- $3^{\prime}$ and reverse $5^{\prime}$-ACTCCAGGTGGGT CATAAGGTTTG-3'.

\subsection{Cellular $\mathrm{NAD}^{+}(\mathrm{H})$ Levels, and Cytoplasmic and Nuclear Extract Preparation}

The cytosolic $\mathrm{NAD}^{+} / \mathrm{NADH}$ ratio was determined using the $\mathrm{NAD}^{+} / \mathrm{NADH}$ Quantitation Colorimetric Kit (BioVision K337-100) as per the manufacturer's instructions. The H9c2 cells were washed twice in ice-cold PBS and detached with PBS. After the removal of the supernatant, cytoplasmic and nuclear proteins were extracted from the cells by means of the Cytoplasmic and Nuclear Protein Extraction Kit (BIOTOOLS Co., Ltd., New Taipei City, Taiwan), according to the manufacturer's instructions.

\subsection{A Chromatin Immunoprecipitation (ChIP) Assay}

ChIP was performed with the SimpleChIP ${ }^{\circledR}$ Enzymatic Chromatin IP Kit (Cell Signaling Technology, Danvers, MA, USA). Briefly, cells were incubated with $1 \%$ formaldehyde at room temperature for $10 \mathrm{~min}$ for cross-linking proteins to DNA. Chromatin was sonicated, of which $10 \mathrm{mg}$ was subjected to immunoprecipitation with antibodies [anti-ATF4 (Santa Cruz Biotechnology, Dallas, TX, USA), anti-ac-H3K9 (Merck, Kenilworth, NJ, USA), or negative control (Normal Mouse IgG; Cell Signaling Technology, Danvers, MA, USA)] at $4{ }^{\circ} \mathrm{C}$ overnight with rotation. The following day, chromatin/antibody complexes were pulled down from the solution by incubation with salmon sperm DNA-saturated $50 \%$ protein A/G-Sepharose beads at $4{ }^{\circ} \mathrm{C}$ for $2 \mathrm{~h}$. The cross-linking was reversed by heating at $65^{\circ} \mathrm{C}$ for $30 \mathrm{~min}$, followed by treatment with $100 \mathrm{~g} / \mathrm{L}$ proteinase $\mathrm{K}$ at $65^{\circ} \mathrm{C}$ for $2 \mathrm{~h}$. DNA purification was performed using the kit mentioned above, and the purified DNA was analyzed by PCR. The primers for CHOP were forward 5'-AAGTTCAGGAAGGACAGCCG-3 ${ }^{\prime}$ and reverse 5'-CGTTATCTCGGACCCGGAAG-3'.

\subsection{Acquisition Systems and Statistical Analysis}

Continuous variables were expressed as the mean \pm standard error of the mean. A Student's $t$-test or Pearson's Chi-square test was performed to evaluate the differences. GraphPad Prism 5 (Systat Software 3.5, Inc., San Jose, CA, USA) was used for statistical comparisons. In figures, " $\mathrm{n}$ " stands for the total number of cells per heart ( $\mathrm{n}=$ cells $/$ hearts), and " $\mathrm{N}$ " is the number of mice. Statistical significance is indicated by ${ }^{*},{ }^{*}$, and ${ }^{* *}$ for $p<0.05, p<0.01$, and $p<0.001$, respectively.

\section{Results}

3.1. The Induction of CHOP and ATF4 Expression in Cardiomyocytes of Mice and H9c2 Cells by HFD and PA, Respectively

The HFD is one of the inducers of ER stress $[19,20]$. To address the role of Sirt1 in HFDinduced ER stress in cardiomyocytes, we fed 8-week-old Sirt1 ${ }^{\mathrm{f} / \mathrm{f}}$ and Sirt1 ${ }^{-/-}$mice with HFD for 8 weeks. CHOP and ATF4 protein levels and mRNA expression were higher in the control mice fed with the HFD compared to those fed the SD (Figure 1A-C). Sirt1 ${ }^{-/-}$mice showed increased protein levels and mRNA expression of CHOP and ATF4 as compared to the control mice fed either the SD or HFD (Figure 1B,C). We exposed H9c2 cells to PA and investigated the expression of CHOP and ATF4. The protein levels and mRNA expression of both ATF4 and CHOP in H9c2 cells were significantly increased in the PA treatment in a dose-dependent manner (Figure 2A-C) and in a time-dependent manner (Figure 2D-F). 
(A)
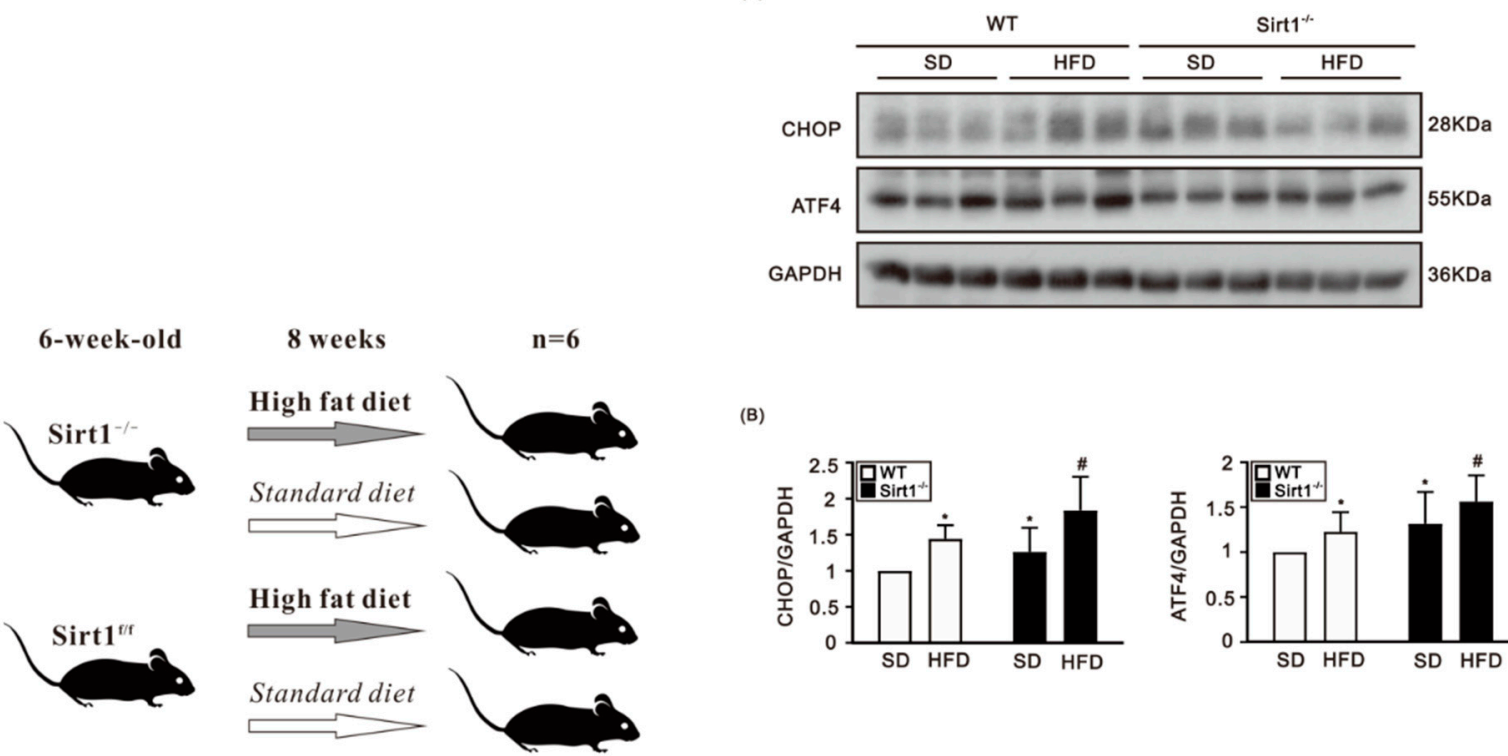

(B)
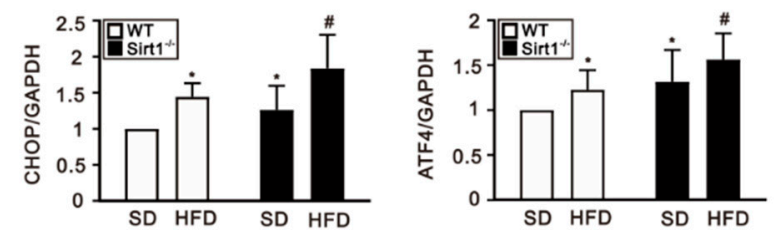

(C)
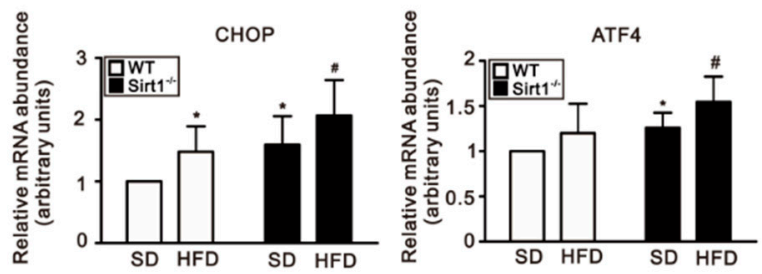

Figure 1. (Left) Scheme of experimental animal usage. (Right) Protein levels and mRNA expression of CHOP and ATF4 in ventricular tissues from control and Sirt1 ${ }^{-/}$mice fed either the SD or HFD. (A) A representative immunoblots of CHOP and ATF4 in control and Sirt1 ${ }^{-/}$- mice fed with either the SD or HFD. (B) The HFD increased protein levels of CHOP and ATF4 in WT mice as compared to those fed with the SD. The protein levels of CHOP and ATF4 were higher in Sirt1 ${ }^{-/-}$mice compared to the control mice fed either the SD or HFD ( $\mathrm{N}=6$ for each group; ${ }^{*}$ compared to WT mice on the $\mathrm{SD}$, \# compared to WT mice on the HFD; * $p<0.05$; \# $p<0.05$ ). (C) Relative mRNA expression of CHOP and ATF4 was higher in Sirt1 ${ }^{-/-}$mice compared to the control mice fed with either the SD or HFD ( $=6$ for each group; * compared to WT mice on the SD, \# compared to WT mice on the HFD; $\left.{ }^{*} p<0.05 ; \# p<0.05\right)$. (SD: standard diet; HFD: high fat diet; WT: wide type).

\subsection{Palmitate Decreases Sirt1's Deacetylating Activity Mediated by the Downregulation of Cytosolic NAD ${ }^{+}$in $\mathrm{H} 9 \mathrm{c} 2$ Cells}

As is known, Sirt1 deacetylates acetyl-lysine in histones, e.g., H3K9Ac, to modulate gene transcription [21]. To investigate the function of Sirt1 under PA-induced ER stress in $\mathrm{H} 9 \mathrm{c} 2$ cells, we quantified Sirt1 protein expression and acetyl-lysine9 in histone H3 (H3K9Ac), a primary target of Sirt1. While the protein level of Sirt1 remained unchanged, the level of H3K9Ac significantly increased in a dose-dependent and time-dependent manner in $\mathrm{H} 9 \mathrm{c} 2$ cells after treatment with PA (see Figure 3A,B for the dose-course experiment and Figure 3C,D for the time-course experiment). The increased amount of H3K9Ac indicated the decreased enzymatic activity of Sirt1 may be not mediated by the downregulation of the Sirt1 protein amount. We next examined whether the cellular $\mathrm{NAD}^{+} / \mathrm{NADH}$ ratio was affected, which may be responsible for the reduced deacetylating activity of Sirt1, under PA-induced ER stress. Our data showed that $\mathrm{NAD}^{+} / \mathrm{NADH}$ ratios significantly decreased in $\mathrm{H} 9 \mathrm{c} 2$ cells treated with PA in a dose-dependent manner (Figure 3E). 
(A)

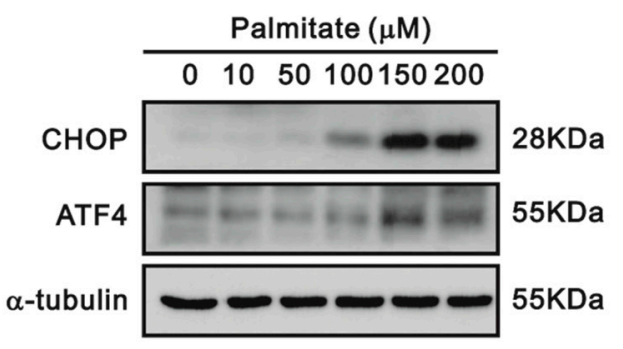

(C)

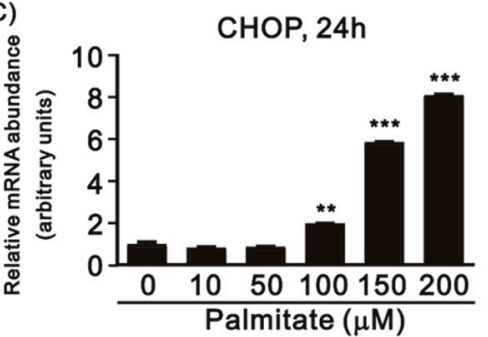

(D)
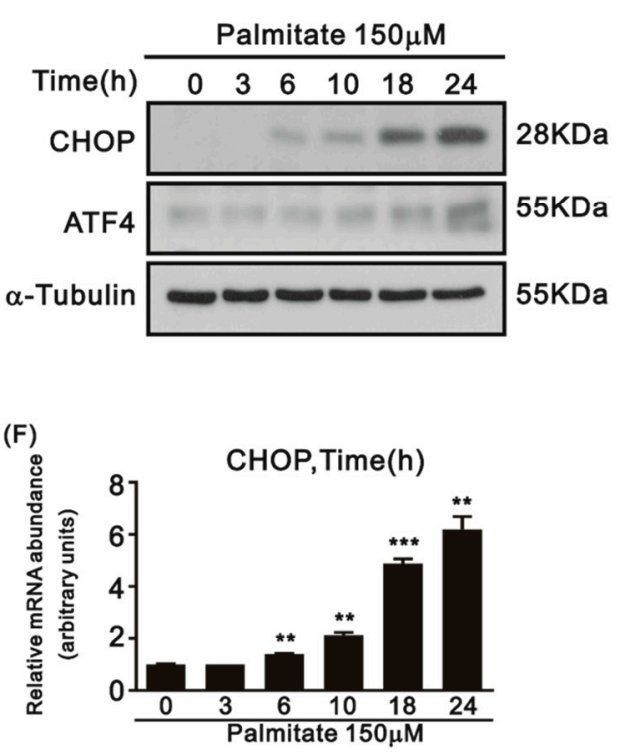

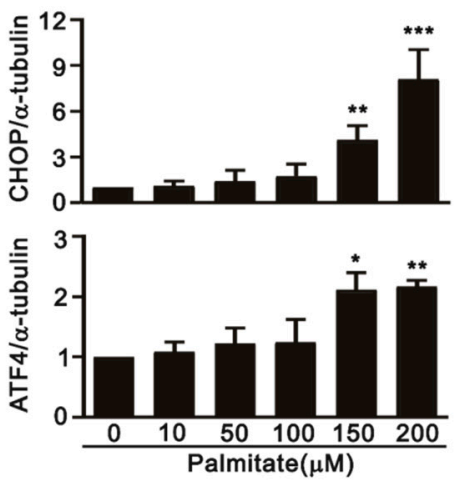

ATF4, 24h

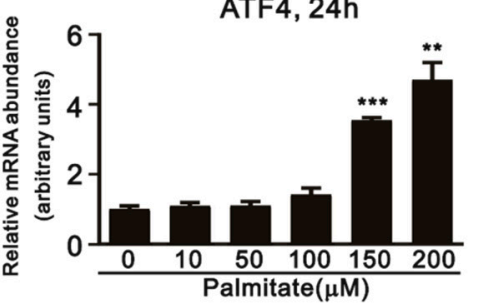

(E)
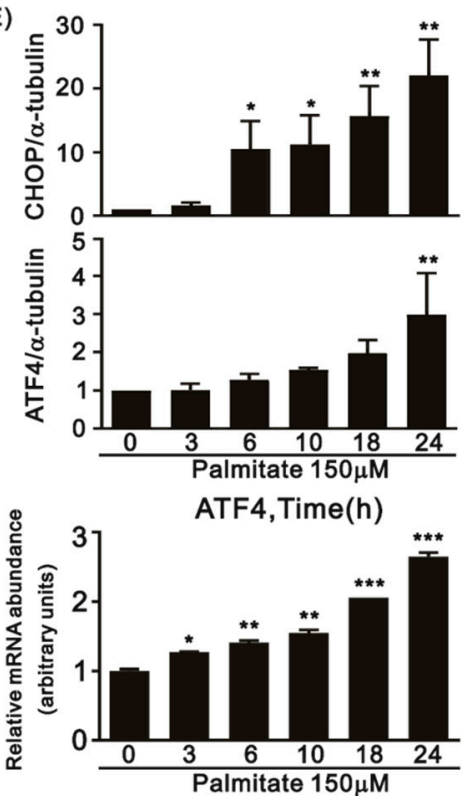

Figure 2. Protein levels and mRNA expression of CHOP and ATF4 in the H9c2 cells treated with PA. (A) A representative immunoblot and (B) mean data for $\mathrm{CHOP}$ and ATF4 in the H9c2 cells treated with PA $(0-200 \mu \mathrm{M})$ at $24 \mathrm{~h}\left(\mathrm{n}=3\right.$; asterisk (s): compared to the $0 \mu \mathrm{M}$ PA control group; ${ }^{*} p<0.05,{ }^{* *} p<0.01$, *** $p<0.001)$. (C) Relative levels of CHOP and ATF4 mRNAs in H9c2 cells after PA $(0-200 \mu \mathrm{M})$ treatment at $24 \mathrm{~h}\left(\mathrm{n}=3\right.$; asterisk (s): compared to the $0 \mu \mathrm{M}$ PA control group; $\left.{ }^{* *} p<0.01,{ }^{* * *} p<0.001\right)$. (D) A representative immunoblot and (E) mean data for CHOP and ATF4 in H9c2 cells treated with PA (150 $\mu \mathrm{M}$, $0-24 \mathrm{~h})\left(\mathrm{n}=3\right.$; asterisk (s): compared to the $0 \mu \mathrm{M}$ PA control group; $\left.{ }^{*} p<0.05,{ }^{* *} p<0.01\right)$. (F) Relative expression levels of $C H O P$ and ATF4 mRNA in H9c2 cells after PA $(150 \mu \mathrm{M}, 0-24 \mathrm{~h})$ treatment $(\mathrm{n}=2$; asterisk (s): compared to the $0 \mu \mathrm{M}$ PA control group; ${ }^{*} p<0.05,{ }^{* *} p<0.01,{ }^{* * *} p<0.001$ ). 
(A)

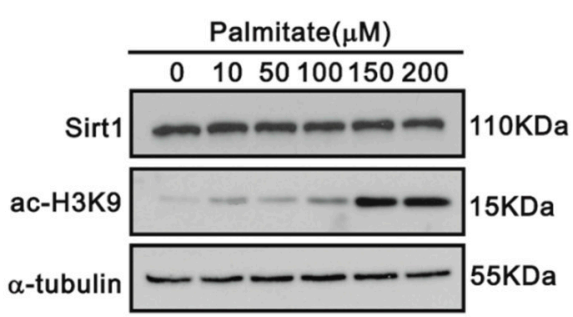

(C)

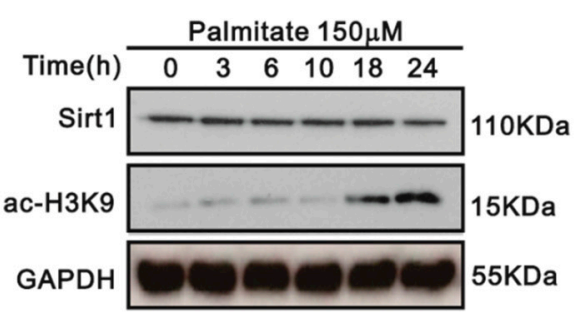

(E)

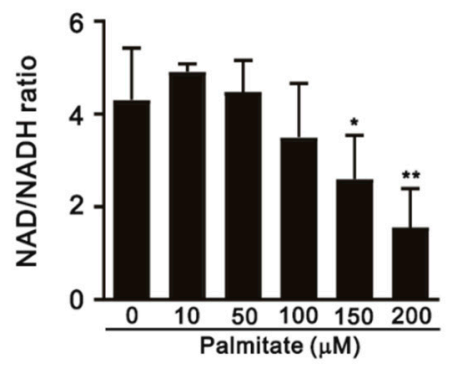

(B)

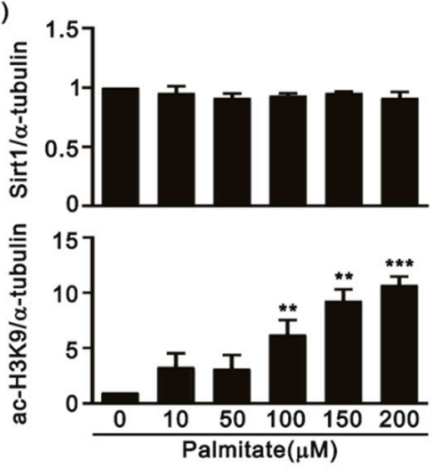

(D)

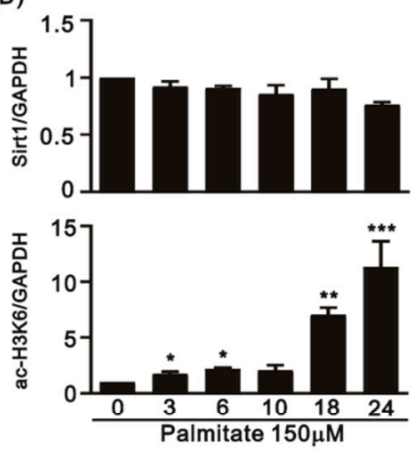

Figure 3. Protein levels of Sirt1, the amount of H3K9Ac, and the cytosolic NAD ${ }^{+} / \mathrm{NADH}$ ratio in H9c2 cells treated with PA. (A) A representative immunoblot and (B) mean data for Sirt1 and H3K9Ac in $\mathrm{H} 9 \mathrm{c} 2$ cells treated with PA $(0-200 \mu \mathrm{M})$ at $24 \mathrm{~h}(\mathrm{n}=3$; asterisk: compared to the $0 \mu \mathrm{M}$ PA control group; $\left.{ }^{* *} p<0.01,{ }^{* * *} p<0.001\right)$. (C) A representative immunoblot and (D) mean data for Sirt1 and H3K9Ac in H9c2 cells treated with PA $(150 \mu \mathrm{M}, 0-24 \mathrm{~h})(\mathrm{n}=3$; asterisk (s): compared to the $0 \mu \mathrm{M}$ PA control group; $\left.{ }^{*} p<0.05,{ }^{* *} p<0.01,{ }^{* * *} p<0.001\right)$. (E) The cytosolic NAD ${ }^{+} / \mathrm{NADH}$ ratio in the H9c2 cells treated with PA decreased in a PA dose-dependent manner $\left(\mathrm{n}=3 ;^{*} p<0.05,{ }^{* *} p<0.01\right)$.

\subsection{Sirt1 Reduces mRNA and Protein Expression of ATF4 and CHOP in H9c2 Cells}

It is important to verify the decreased ratio of $\mathrm{NAD}^{+}$to NADH to assess Sirt1's deacetylating enzymatic activity. One dominant negative Sirt1 mutant (H363Y) has been found to lose its deacetylating activity [22]. Hence, we suppressed the enzymatic activity of Sirt1 via this dominant negative H363Y mutation in H9c2 cells to determine whether the abrogation of Sirt1 enzymatic activity affects the expression of CHOP and ATF4. Sirt1 H363Y induced mRNA and protein expression of CHOP and ATF4 in H9c2 cells (Figure 4A,B). After PA treatment, the protein expression of CHOP and ATF4 increased in Sirt1 H363Ytransfected H9c2 cells (Figure 4C,D). On the contrary, H3K9Ac upregulation by Sirt1 H363Y was attenuated by the PA treatment. 
(A)

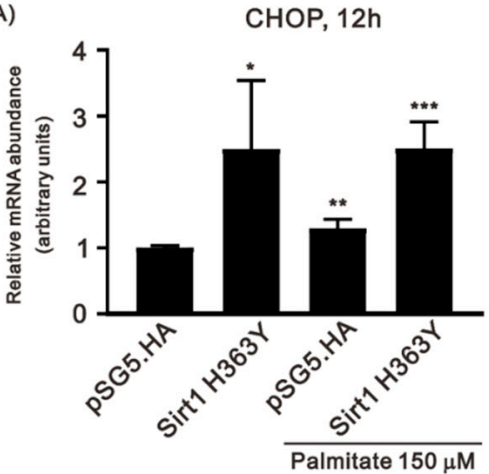

(C)

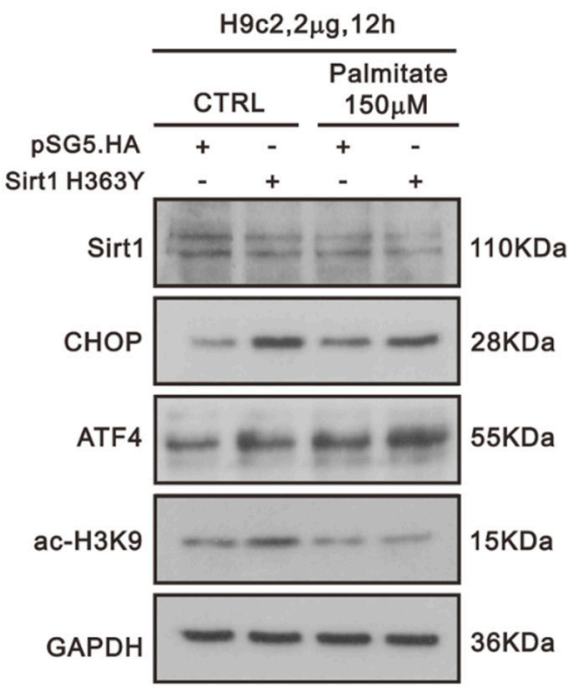

(B)

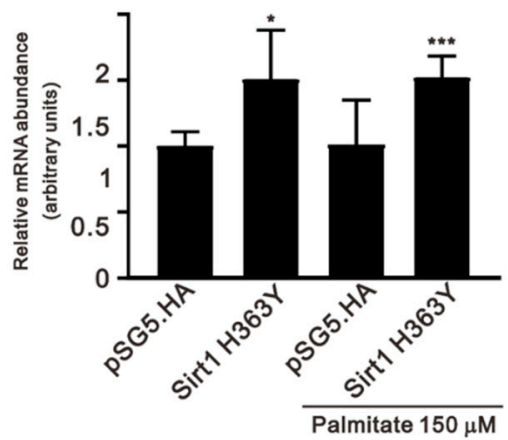

(D)

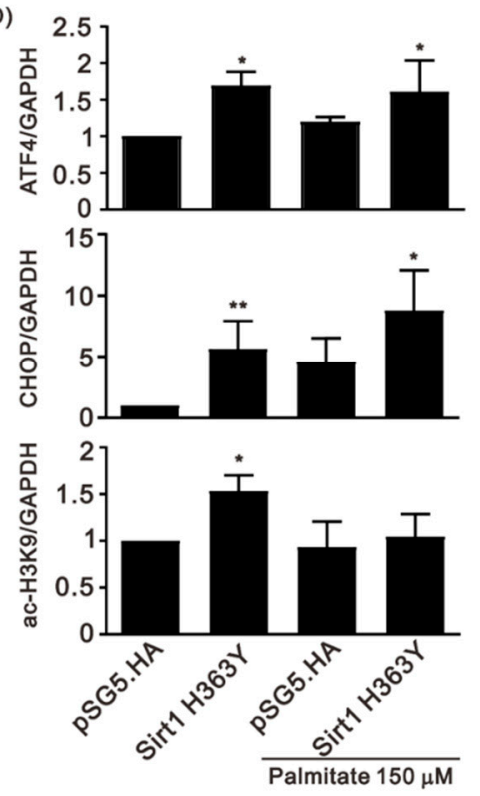

Figure 4. Effects of transfection with the plasmid encoding the Sirt1 H363Y mutant in H9c2 cells. $(\mathbf{A}, \mathbf{B})$ Relative mRNA expression of CHOP and ATF4 in pSG5.HA- or H363Y mutant Sirt1 plasmidtransfected H9c2 cells treated or not treated with PA ( $n=3$; asterisk (s): compared to the pSG5.HA group; ${ }^{*} p<0.05,{ }^{* *} p<0.01$, and $\left.{ }^{* *} p<0.001\right)$. (C,D) The protein expression of CHOP and ATF4 in pSG5.HA- or H363Y mutant Sirt1 plasmid-transfected H9c2 cells treated or not treated with PA ( $\mathrm{n}=3$; asterisk (s): compared to the pSG5.HA group; ${ }^{*} p<0.05$ and ${ }^{* *} p<0.01$ ).

\subsection{SRT1720 Enhances Nuclear Translocation of ATF4 and Reduces Histone H3K9 Acetylation in the CHOP Promoter}

The above-mentioned data indicated that PA reduces the cytosolic NAD ${ }^{+}$amount, thereby suppressing the enzymatic activity of Sirt1 in H9c2 cells. Accordingly, we applied a Sirt1 activator, SRT1720, to address the role of the deacetylating activity of Sirt1 in the regulation of ATF4 and the CHOP expression in H9c2 cells. Furthermore, SRT1720 did not suppress mRNA expression of CHOP and ATF4 in PA-treated H9c2 cells (Figure 5A). SRT1720 reduced the levels of H3K9Ac in PA-treated H9c2 cells, suggesting that it is an activator of Sirt1's deacetylating activity (Figure 5B). Under ER stress, ATF4 may migrate to the nucleus, bind to target genes, and regulate their transcription [23]. Next, we tested whether SRT1720 affects the cytosolic and nuclear fractions of Sirt1 and ATF4 in H9c2 cells with or without PA. Nuclear translocation of ATF4 in H9c2 cells was increased by the PA treatment and further strengthened when combined with SRT1720 treatment (Figure 5C,D). Neither PA nor SRT1720 influenced the cytosolic and nuclear fractions of Sirt1 (Figure 5C,D). The ChIP results showed that in H9c2 cells, PA did not affect the recruitment of ATF4 to the CHOP promoter (Figure 5E,F). SRT1720 did not have effect on the amount of H3K9Ac in the CHOP promoter in PA-treated H9c2 cells (Figure 5E,F). 
(A)

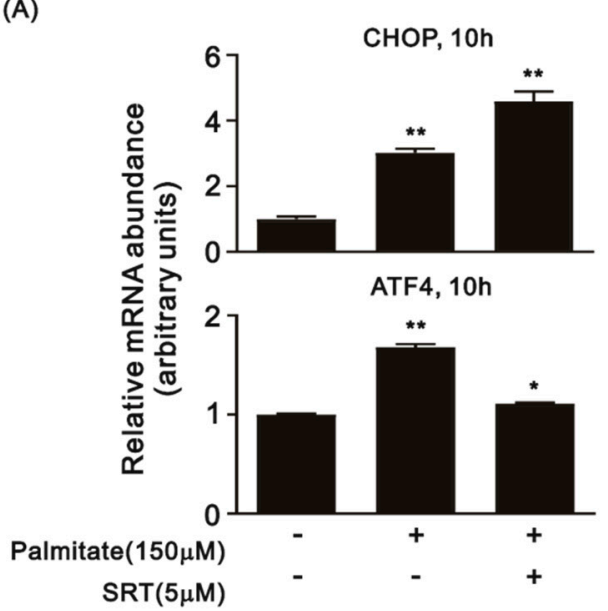

(C)

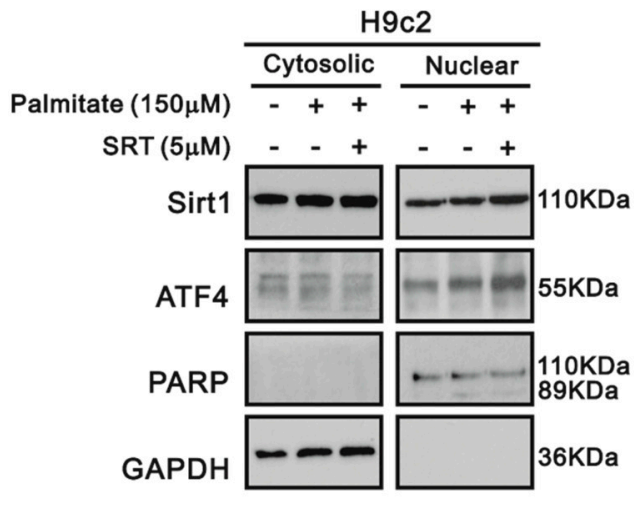

(E)

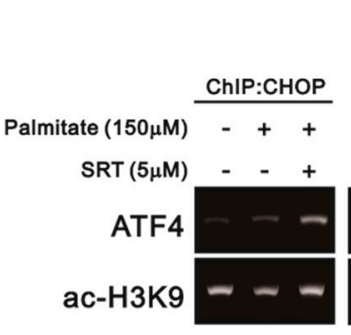

(B)

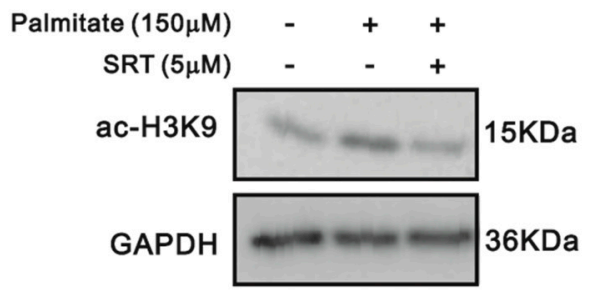

(D)

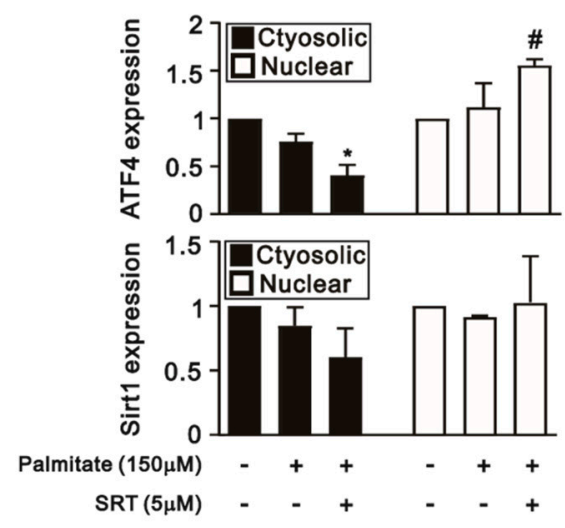

(F)

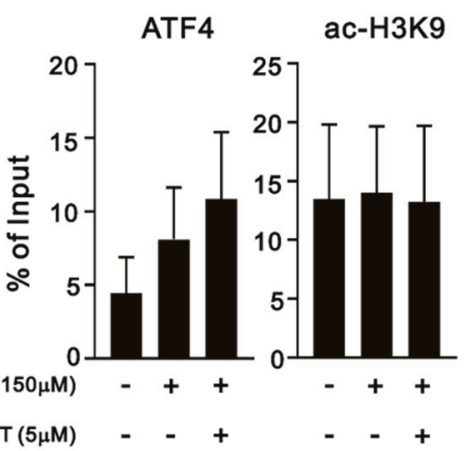

Figure 5. Effects of SRT1720 on CHOP and ATF4 in the H9c2 cells treated with PA. (A) The relative mRNA expression of CHOP in PA-treated H9c2 cells with or without SRT1720 ( $\mathrm{n}=3 ;{ }^{*} p<0.05$, ** $p<0.01)$. (B) A representative immunoblots of ATF4 and H3K9Ac in PA-treated H9c2 cells with or without SRT1720 ( $n=3)$. (C,D) The expression of ATF4 and Sirt1 in cytosolic and nuclear fractions ( $\mathrm{n}=3$; $^{*}$ compared to the cytosol control; \# compared to the nuclear control; ${ }^{*} p<0.05, \# p<0.05$ ). (E,F) ChIP for assessing the interaction between ATF4 and the CHOP promotor and between H3K9Ac and the CHOP promotor in PA-treated H9c2 cells with or without SRT1720 (n=3).

\section{Discussion}

Lipotoxicity is an important contributor to cardiac dysfunction in obesity associated heart disease [24,25]. An excessive uptake of fatty acids can result in either enhanced oxidation or abnormal accumulation of toxic lipid species such as ceramides and diacylglycerides, causing lipotoxicity in the heart and other solid organs, and the one of crucial underlying mechanism is ER stress and impaired UPR signaling [26]. Some evidence indicates that the activation of Sirt1/AMPK signaling may prevent cells from fatty acid-induced oxidative 
stress and inflammation [27], but the participation of Sirt1 in lipotoxicity-induced ER stress remains unclear. In the present study, we utilized mice fed a palmitate-enriched HFD and palmitate-treated $\mathrm{H} 9 \mathrm{c} 2$ cells as in vivo and in vitro models of lipotoxicity and examined the cellular consequences, including ER stress and Sirt1 activity, as well as the crosstalk between these cellular phenomena.

There are studies showing that the lipotoxicity associated with ER stress increases ATF4 and CHOP mRNA expression, but the underlying mechanism still needs to be clarified $[26,28,29]$. Our data reveal that palmitate induces the expression of ER stress markers CHOP and ATF4 in vivo and in vitro, in line with other reports [26,28,29]. One study suggested that palmitate-induced cardiomyocyte dysfunction is mediated by ER stress and thereby promotes cell death [5]. Furthermore, Sirt1 may confer cardio-protection against ER stress. Alexandre et al. have reported that cardiac Sirt1 deficiency increases the contractile dysfunction caused by ER stress in a Sirt1 knockout mouse model, and the mechanism may involve eIF2 $\alpha$ deacetylation [11]. According to our results, Sirt1 ${ }^{-/-}$ cardiomyocytes show higher expression of CHOP and ATF4 as compared to cardiomyocytes from the control mice fed either the SD or HFD, also suggesting a protective role of Sirt1 against HFD-induced ER stress in cardiomyocytes.

As an $\mathrm{NAD}^{+}$-dependent reaction, the protein deacetylation catalyzed by Sirt1 is accompanied by the hydrolysis of $\mathrm{NAD}^{+}$. Therefore, we propose that the decreased Sirt1 activity is associated with reduced $\mathrm{NAD}^{+}$concentration in palmitate-treated $\mathrm{H} 9 \mathrm{c} 2$ cells, in line with other reports $[30,31]$. Some research has revealed that changes in cytosolic $\mathrm{NAD}^{+}$levels alter Sirt1 activity $[12,32,33]$. Additionally, one report has shown that a reduced cellular NAD ${ }^{+}$ concentration, resulting from the conversion of $\mathrm{NAD}^{+}$to NADH by the glucose metabolic pathway, leads to lower Sirt1 activity [34]. As a consequence of its dependence on $\mathrm{NAD}^{+}$and therefore on the cellular $\mathrm{NAD}^{+} / \mathrm{NADH}$ ratio, Sirt1 has emerged as a key metabolic sensor with respect to various tissues [35]. There is evidence [36,37] that in a high-energy state, such as that associated with an HFD and obesity, Sirt1 activity may decline with a decreased level of $\mathrm{NAD}^{+}$. Therefore, we suggest that palmitate-induced ER stress diminishes the cytosolic level of $\mathrm{NAD}^{+}$, which in turn reduces Sirt1 enzymatic activity.

Our results indicate that the dominant negative Sirt1 H363Y mutant increases the expression of ATF4 and CHOP, also implying a protective role of Sirt1 (via its enzymatic activity) against ER stress [11]. Nevertheless, PA did not raise either ATF4 or CHOP expression in the H9c2 cells transfected with the plasmid encoding the Sirt1 H363Y mutant, and the same was true for H3K9Ac. We believe that the plasmid transfection of H9c2 cells attenuates the influence of PA or PA weakens the effects of the plasmid transfection. In addition, our data indicate that autophagy-related proteins beclin 1 and p63 are upregulated in the H9c2 cells treated with PA (Supplemental Figure S1). PA-induced autophagy has been shown to abrogate the partial apoptosis caused by PA [38]. Therefore, ATF4 or CHOP expression in the H9c2 cells transfected with the Sirt1 H363Y mutant may be abrogated by the autophagy induced by PA. The increase in the H3K9Ac level in PA-treated H9c2 cells was attenuated by SRT1720 treatment, implying an increased deacetylation function of Sirt1, although the $\mathrm{NAD}^{+}$concentration was not restored under these conditions. On the other hand, the PA-induced expression of ATF4 was not weakened by SRT1720 treatment. Furthermore, our findings show that the translocation of ATF4 to the nucleus increases upon SRT1720 treatment; therefore, the increased nuclear recruitment may enhance the gene regulatory function of ATF4. The amount of binding between ATF4 and the CHOP promoter did not affect with PA treatment. With PA plus SRT1720 treatment, the extent of binding between ATF4 and the CHOP promoter did not increase, although SRT1720 led to more ATF4 becoming available in the nucleus. Additionally, SRT1720 strengthened the activity of Sirt1 but only had a limited impact on the CHOP and ATF4 expression induced by PA treatment, suggesting the involvement of more than a deacetylating activity. The protein-protein interaction and nuclear localization are being researched in more detail to decipher the current complicated findings. 
As a possible metabolic sensor, the activity of Sirt1 and the level of $\mathrm{NAD}^{+}$could be used for assessment of ER stress. Therefore, restoring the activity of Sirt1 and the level of $\mathrm{NAD}^{+}$could provide clinically therapeutic potential for protecting various organs such as the heart from ER stress-induced injury [39]. These findings may also provide potential clinical application for Sirt1 in the treatment of metabolic syndrome associated cardiovascular disease. Future studies on Sirt1 overexpression using plasmid transfection could be conducted to examine if increased amount of Sirt1 provides protection against PA-induced ER stress via post-translational modification of target substrates. Whether Sir1 has protective role in PA-induced ER stress associated endothelial dysfunction may be another future direction to be investigated.

In conclusion, PA-induced ER stress is mediated through the upregulation of ATF4 and $\mathrm{CHOP}$ mRNAs and proteins. PA reduces the amount of cytosolic $\mathrm{NAD}^{+}$, which in turn suppresses Sirt1 activity. Nevertheless, the Sirt1 activator SRT1720 does not attenuate the expression of CHOP and ATF4 induced by PA, but enhances the nuclear translocation of ATF4.

Supplementary Materials: The following supporting information can be downloaded at: https: / /www.mdpi.com/article/10.3390/life12020182/s1, Figure S1: Protein levels of autophagy-related proteins in the $\mathrm{H} 9 \mathrm{c} 2$ cells treated with PA.

Author Contributions: H.-Y.Y., J.-Y.C. and P.-L.Y. conducted the experiments; H.-Y.Y., J.-Y.C., Y.-N.H., P.-Z.L. and S.-C.H. analyzed the data. H.-Y.Y., Y.-N.H., S.-C.H. and S.-M.H. interpreted the results of the experiments; J.-Y.C. and P.-L.Y. prepared the figures; H.-Y.Y. drafted the manuscript; H.-Y.Y. and S.-M.H. edited and revised the manuscript; C.-S.T. and C.-Y.L. conceived and designed the study. All authors have read and agreed to the published version of the manuscript.

Funding: This work was supported by Ministry of Science and Technology of Taiwan (MOST-1072314-B-016-063-MY3 and MOST108-2314-B-016-050); Veterans General Hospital at Taipei Taichung and Kaohsiung, Tri-Service General Hospital, and Academia Sinica Joint Research Program (VTA109V1-3-2); Tri-Service General Hospital, Taiwan (TSGH-C01-110018, TSGH-E-109209, TSGH-C05-111043, TSGH-C05-111042). Ministry of National Defense-Medical Affairs Bureau (MND-MAB-D11129 and MND-MAB-110-018).

Institutional Review Board Statement: The animal study protocol was conducted with the approval of the Institutional Animal Care and Use Committee (IACUC, permit No. 19-364) of the National Defense Medical Center (Taipei, Taiwan) and in accordance with the National Institutes of Health guidelines.

Informed Consent Statement: Not applicable.

Data Availability Statement: The data of the present study are available from the corresponding authors upon reasonable request.

Conflicts of Interest: The authors declare no conflict of interest.

\section{References}

1. Nakamura, M.; Sadoshima, J. Cardiomyopathy in obesity, insulin resistance and diabetes. J. Physiol. 2020, 598, $2977-2993$. [CrossRef] [PubMed]

2. MacDonald-Ramos, K.; Martínez-Ibarra, A.; Monroy, A.; Miranda-Ríos, J.; Cerbón, M. Effect of Dietary Fatty Acids on MicroRNA Expression Related to Metabolic Disorders and Inflammation in Human and Animal Trials. Nutrients 2021, 13, 1830. [CrossRef] [PubMed]

3. Wali, J.A.; Jarzebska, N.; Raubenheimer, D.; Simpson, S.J.; Rodionov, R.N.; O'Sullivan, J.F. Cardio-Metabolic Effects of High-Fat Diets and Their Underlying Mechanisms-A Narrative Review. Nutrients 2020, 12, 1505. [CrossRef] [PubMed]

4. Drosatos, K.; Schulze, P.C. Cardiac lipotoxicity: Molecular pathways and therapeutic implications. Curr. Heart Fail. Rep. 2013, 10, 109-121. [CrossRef] [PubMed]

5. Yang, L.; Guan, G.; Lei, L.; Liu, J.; Cao, L.; Wang, X. Oxidative and endoplasmic reticulum stresses are involved in palmitic acid-induced H9c2 cell apoptosis. Biosci. Rep. 2019, 39, BSR20190225. [CrossRef] [PubMed]

6. Xu, C.; Bailly-Maitre, B.; Reed, J.C. Endoplasmic reticulum stress: Cell life and death decisions. J. Clin. Investig. 2005, 115, 2656-2664. [CrossRef] [PubMed]

7. Matsushima, S.; Sadoshima, J. The role of sirtuins in cardiac disease. Am. J. Physiol. Heart Circ. Physiol. 2015, 309, H1375-H1389. [CrossRef] 
8. Sanz, M.N.; Grimbert, L.; Moulin, M.; Gressette, M.; Rucker-Martin, C.; Lemaire, C.; Mericskay, M.; Veksler, V.; Ventura-Clapier, R.; Garnier, A.; et al. Inducible Cardiac-Specific Deletion of Sirt1 in Male Mice Reveals Progressive Cardiac Dysfunction and Sensitization of the Heart to Pressure Overload. Int. J. Mol. Sci. 2019, 20, 5005. [CrossRef] [PubMed]

9. Gorski, P.A.; Jang, S.P.; Jeong, D.; Lee, A.; Lee, P.; Oh, J.G.; Chepurko, V.; Yang, D.K.; Kwak, T.H.; Eom, S.H.; et al. Role of SIRT1 in Modulating Acetylation of the Sarco-Endoplasmic Reticulum Ca $\left.{ }^{2+}\right)$-ATPase in Heart Failure. Circ. Res. 2019, 124, e63-e80. [CrossRef]

10. Bugyei-Twum, A.; Ford, C.; Civitarese, R.; Seegobin, J.; Advani, S.L.; Desjardins, J.F.; Kabir, G.; Zhang, Y.; Mitchell, M.; Switzer, J.; et al. Sirtuin 1 activation attenuates cardiac fibrosis in a rodent pressure overload model by modifying Smad2/3 transactivation. Cardiovasc. Res. 2018, 114, 1629-1641. [CrossRef]

11. Prola, A.; Pires Da Silva, J.; Guilbert, A.; Lecru, L.; Piquereau, J.; Ribeiro, M.; Mateo, P.; Gressette, M.; Fortin, D.; Boursier, C.; et al. SIRT1 protects the heart from ER stress-induced cell death through eIF2 $\alpha$ deacetylation. Cell Death Differ. 2017, 24, 343-356. [CrossRef] [PubMed]

12. Li, J.; Dou, X.; Li, S.; Zhang, X.; Zeng, Y.; Song, Z. Nicotinamide ameliorates palmitate-induced ER stress in hepatocytes via cAMP/PKA/CREB pathway-dependent Sirt1 upregulation. Biochim. Biophys. Acta 2015, 1853, 2929-2936. [CrossRef]

13. Hsu, Y.J.; Hsu, S.C.; Hsu, C.P.; Chen, Y.H.; Chang, Y.L.; Sadoshima, J.; Huang, S.M.; Tsai, C.S.; Lin, C.Y. Sirtuin 1 protects the aging heart from contractile dysfunction mediated through the inhibition of endoplasmic reticulum stress-mediated apoptosis in cardiac-specific Sirtuin 1 knockout mouse model. Int. J. Cardiol. 2017, 228, 543-552. [CrossRef] [PubMed]

14. Weng, C.H.; Chung, F.P.; Chen, Y.C.; Lin, S.F.; Huang, P.H.; Kuo, T.B.; Hsu, W.H.; Su, W.C.; Sung, Y.L.; Lin, Y.J.; et al. Pleiotropic Effects of Myocardial MMP-9 Inhibition to Prevent Ventricular Arrhythmia. Sci. Rep. 2016, 6, 38894. [CrossRef] [PubMed]

15. Lin, C.Y.; Hsu, Y.J.; Hsu, S.C.; Chen, Y.; Lee, H.S.; Lin, S.H.; Huang, S.M.; Tsai, C.S.; Shih, C.C. CB1 cannabinoid receptor antagonist attenuates left ventricular hypertrophy and Akt-mediated cardiac fibrosis in experimental uremia. J. Mol. Cell. Cardiol. 2015, 85, 249-261. [CrossRef]

16. Hsu, S.C.; Huang, S.M.; Chen, A.; Sun, C.Y.; Lin, S.H.; Chen, J.S.; Liu, S.T.; Hsu, Y.J. Resveratrol increases anti-aging Klotho gene expression via the activating transcription factor 3/c-Jun complex-mediated signaling pathway. Int. J. Biochem. Cell Biol. 2014, 53, 361-371. [CrossRef]

17. Brunet, A.; Sweeney, L.B.; Sturgill, J.F.; Chua, K.F.; Greer, P.L.; Lin, Y.; Tran, H.; Ross, S.E.; Mostoslavsky, R.; Cohen, H.Y.; et al. Stress-dependent regulation of FOXO transcription factors by the SIRT1 deacetylase. Science 2004, 303, 2011-2015. [CrossRef]

18. Amat, R.; Planavila, A.; Chen, S.L.; Iglesias, R.; Giralt, M.; Villarroya, F. SIRT1 controls the transcription of the peroxisome proliferator-activated receptor-gamma Co-activator-1alpha (PGC-1alpha) gene in skeletal muscle through the PGC-1alpha autoregulatory loop and interaction with MyoD. J. Biol. Chem. 2009, 284, 21872-21880. [CrossRef]

19. Hsu, H.C.; Chen, C.Y.; Lee, B.C.; Chen, M.F. High-fat diet induces cardiomyocyte apoptosis via the inhibition of autophagy. Eur. J. Nutr. 2016, 55, 2245-2254. [CrossRef]

20. Tan, L.; Harper, L.; McNulty, M.A.; Carlson, C.S.; Yammani, R.R. High-fat diet induces endoplasmic reticulum stress to promote chondrocyte apoptosis in mouse knee joints. FASEB J. Off. Publ. Fed. Am. Soc. Exp. Biol. 2020, 34, 5818-5826. [CrossRef]

21. Yang, G.; Weng, X.; Zhao, Y.; Zhang, X.; Hu, Y.; Dai, X.; Liang, P.; Wang, P.; Ma, L.; Sun, X.; et al. The histone H3K9 methyltransferase SUV39H links SIRT1 repression to myocardial infarction. Nat. Commun. 2017, 8, 14941. [CrossRef] [PubMed]

22. Suter, M.A.; Chen, A.; Burdine, M.S.; Choudhury, M.; Harris, R.A.; Lane, R.H.; Friedman, J.E.; Grove, K.L.; Tackett, A.J.; Aagaard, K.M. A maternal high-fat diet modulates fetal SIRT1 histone and protein deacetylase activity in nonhuman primates. FASEB J. Off. Publ. Fed. Am. Soc. Exp. Biol. 2012, 26, 5106-5114. [CrossRef]

23. Han, J.; Back, S.H.; Hur, J.; Lin, Y.H.; Gildersleeve, R.; Shan, J.; Yuan, C.L.; Krokowski, D.; Wang, S.; Hatzoglou, M.; et al ER-stress-induced transcriptional regulation increases protein synthesis leading to cell death. Nat. Cell Biol. 2013, 15, 481-490. [CrossRef] [PubMed]

24. Leroy, C.; Tricot, S.; Lacour, B.; Grynberg, A. Protective effect of eicosapentaenoic acid on palmitate-induced apoptosis in neonatal cardiomyocytes. Biochim. Biophys. Acta 2008, 1781, 685-693. [CrossRef] [PubMed]

25. Wende, A.R.; Abel, E.D. Lipotoxicity in the heart. Biochim. Biophys. Acta 2010, 1801, 311-319. [CrossRef] [PubMed]

26. Han, J.; Kaufman, R.J. The role of ER stress in lipid metabolism and lipotoxicity. J. Lipid Res. 2016, 57, 1329-1338. [CrossRef]

27. Xu, H.; Chen, G.F.; Ma, Y.S.; Zhang, H.W.; Zhou, Y.; Liu, G.H.; Chen, D.Y.; Ping, J.; Liu, Y.H.; Mou, X.; et al. Hepatic Proteomic Changes and Sirt1/AMPK Signaling Activation by Oxymatrine Treatment in Rats with Non-alcoholic Steatosis. Front. Pharmacol. 2020, 11, 216. [CrossRef]

28. Fusakio, M.E.; Willy, J.A.; Wang, Y.; Mirek, E.T.; Al Baghdadi, R.J.; Adams, C.M.; Anthony, T.G.; Wek, R.C. Transcription factor ATF4 directs basal and stress-induced gene expression in the unfolded protein response and cholesterol metabolism in the liver. Mol. Biol. Cell 2016, 27, 1536-1551. [CrossRef]

29. Wortel, I.M.N.; van der Meer, L.T.; Kilberg, M.S.; van Leeuwen, F.N. Surviving Stress: Modulation of ATF4-Mediated Stress Responses in Normal and Malignant Cells. Trends Endocrinol. Metab. TEM 2017, 28, 794-806. [CrossRef]

30. Flores-León, M.; Pérez-Domínguez, M.; González-Barrios, R.; Arias, C. Palmitic Acid-Induced NAD $\left({ }^{+}\right)$Depletion is Associated with the Reduced Function of SIRT1 and Increased Expression of BACE1 in Hippocampal Neurons. Neurochem. Res. 2019, 44, 1745-1754. [CrossRef]

31. Shen, C.; Dou, X.; Ma, Y.; Ma, W.; Li, S.; Song, Z. Nicotinamide protects hepatocytes against palmitate-induced lipotoxicity via SIRT1-dependent autophagy induction. Nutr. Res. 2017, 40, 40-47. [CrossRef] [PubMed] 
32. Cantó, C.; Gerhart-Hines, Z.; Feige, J.N.; Lagouge, M.; Noriega, L.; Milne, J.C.; Elliott, P.J.; Puigserver, P.; Auwerx, J. AMPK regulates energy expenditure by modulating NAD ${ }^{+}$metabolism and SIRT1 activity. Nature 2009, 458, 1056-1060. [CrossRef] [PubMed]

33. Yoshino, J.; Mills, K.F.; Yoon, M.J.; Imai, S. Nicotinamide mononucleotide, a key $\left.\mathrm{NAD}^{+}\right)$intermediate, treats the pathophysiology of diet- and age-induced diabetes in mice. Cell Metab. 2011, 14, 528-536. [CrossRef] [PubMed]

34. Cantó, C.; Menzies, K.J.; Auwerx, J. NAD( $\left(^{+}\right)$Metabolism and the Control of Energy Homeostasis: A Balancing Act between Mitochondria and the Nucleus. Cell Metab. 2015, 22, 31-53. [CrossRef]

35. Anderson, K.A.; Madsen, A.S.; Olsen, C.A.; Hirschey, M.D. Metabolic control by sirtuins and other enzymes that sense NAD $\left.{ }^{+}\right)$, NADH, or their ratio. Biochim. Biophys. Acta Bioenerg. 2017, 1858, 991-998. [CrossRef]

36. Li, X. SIRT1 and energy metabolism. Acta Biochim. Biophys. Sin. 2013, 45, 51-60. [CrossRef]

37. Schug, T.T.; Li, X. Sirtuin 1 in lipid metabolism and obesity. Ann. Med. 2011, 43, 198-211. [CrossRef]

38. Jiang, X.S.; Chen, X.M.; Wan, J.M.; Gui, H.B.; Ruan, X.Z.; Du, X.G. Autophagy Protects against Palmitic Acid-Induced Apoptosis in Podocytes in vitro. Sci. Rep. 2017, 7, 42764. [CrossRef]

39. Wang, F.; Yao, S.; Xia, H. SIRT1 is a key regulatory target for the treatment of the endoplasmic reticulum stress-related organ damage. Biomed. Pharmacother. Biomed. Pharmacother. 2020, 130, 110601. [CrossRef] 